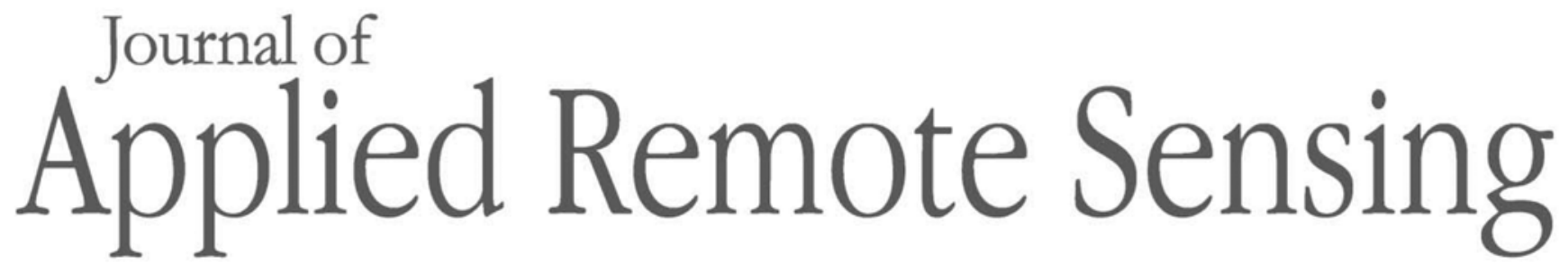

RemoteSensing.SPIEDigitalLibrary.org

\title{
Mineral discrimination by combination of multispectral image and surrounding hyperspectral image
}

\author{
Akihiro Hirai \\ Hideyuki Tonooka
}




\title{
Mineral discrimination by combination of multispectral image and surrounding hyperspectral image
}

\author{
Akihiro Hirai and Hideyuki Tonooka* \\ Ibaraki University, Graduate School of Science and Engineering, Hitachi, Japan
}

\begin{abstract}
A hyperspectral (HS) imager is more effective than a multispectral (MS) imager in mineral discrimination, but spatial coverage of HS images is limited in comparison to that of MS images. Thus Kruse and Perry have proposed a method that uses coincident HS imaging and MS imaging data to extend mineral mapping to larger areas. We propose a method modified from the Kruse and Perry's (K\&P) method. Though the K\&P method derives the MS-based endmember spectra by weighting the HS-based endmember spectra with the response functions of the MS sensor bands, the proposed method obtains the MS-based endmember spectra from surface reflectance spectra of the MS pixels at the same positions with the HS pixels selected as the HS-based endmembers in the overlapping area. The validation study using airborne visible/infrared imaging spectrometer and advanced spaceborne thermal emission and reflection radiometer images over Cuprite and Goldfield areas, Nevada, USA, demonstrates that the proposed method is more robust against spectral inconsistency between the HS- and the MS-images caused by calibration and/or atmospheric correction errors than the K\&P method, though the proposed method is more sensitive to co-registration errors between the HS- and the MS-images than the K\&P method. (C) The Authors. Published by SPIE under a Creative Commons Attribution 4.0 Unported License. Distribution or reproduction of this work in whole or in part requires full attribution of the original publication, including its DOI. [DOI: 10.1117/1.JRS.13.024517]
\end{abstract}

Keywords: mineral mapping; hyperspectral imaging; multispectral imaging; airborne visible/ infrared imaging spectrometer; advanced spaceborne thermal emission and reflection radiometer.

Paper 180964 received Dec. 8, 2018; accepted for publication Apr. 23, 2019; published online May 13, 2019.

\section{Introduction}

A multispectral (MS) imager like the advanced spaceborne thermal emission and reflection radiometer (ASTER) onboard the Terra satellite is an effective tool for mineral mapping, ${ }^{1-5}$ but can perform less well in discriminating some minerals due to various limitations particularly in the number of spectral bands and bandwidth. For example, Rowan and Mars ${ }^{3}$ reported that calcite and dolomite were not successfully discriminated in mineral mapping by orthogonal transformation and interband operation with ASTER data, and Tanaka et al. ${ }^{4}$ reported that kaolinite and dickite were not successfully separated by spectral pattern analysis of ASTER data. A hyperspectral (HS) imager, however, allows us to discriminate minerals more effectively than an MS imager due to advantages offered by the number and narrowness of spectral bands, though these bands have considerable amounts of redundancy in general. For example, Kruse et al. ${ }^{6}$ demonstrated that kaolinite and dickite were successfully discriminated using the airborne visible/infrared imaging spectrometer (AVIRIS). van der Meer et al. compared two methods of Wavelength Mapper and QuanTools for deriving spectral features of minerals from HS images and showed that the results by the two methods were comparable and reproducible. ${ }^{7}$ Kereszturi et al. ${ }^{8}$ detected lithologies and mineral alternations on the surface after the 2012 Te Maari eruptions using airborne HS sensor and terrain models derived by light detection and ranging. Liu et al. ${ }^{9}$ detected muscovite, kaolinite, chlorite, epidote, calcite, and dolomite from Tiangong-1 hyperspectral imager (HIS) data acquired over their study area in China.

On the other hand, HS satellite imagers often sacrifice the spatial resolution and/or the swath width of an acquired image for many spectral bands due to some limitations like satellite to

*Address all correspondence to Hideyuki Tonooka, E-mail: hideyuki.tonooka.dr@vc.ibaraki.ac.jp 
ground communication bandwidth, e.g., the spatial resolution and the swath width of Hyperion onboard the EO-1 satellite are $30 \mathrm{~m}$ and $7.6 \mathrm{~km}$, respectively, ${ }^{10}$ those of the Environmental Mapping and Analysis Program (EnMAP) are $30 \mathrm{~m}$ and $30 \mathrm{~km}$, respectively, ${ }^{11}$ those of the Precursore Iperspettrale della Missione Applicativa (PRISMA) mission are $30 \mathrm{~m}$ and $30 \mathrm{~km}$, respectively, ${ }^{12}$ and those of Hyperspectral Imager Suite (HISUI) on the International Space Station (ISS) are $20 \mathrm{~m}$ (cross track) or $30 \mathrm{~m}$ (along track), and $20 \mathrm{~km}$, respectively. ${ }^{13}$ Since this limitation results in reducing the observation frequency, imaging coverage achieved through a mission period is often much smaller in HS satellite sensor projects than in MS satellite sensor projects, leaving gaps in data coverage. Particularly, HISUI onboard the ISS is predicted to leave many gaps after the mission period of three years, because it has no pointing function and its viewing direction is fixed to the nadir.

An effective approach to such a situation will be to compensate a gap of an HS image using an MS image type that is widely available. Kruse and Perry ${ }^{14,15}$ have developed a method that uses coincident HS imaging and MS imaging data to extend mineral mapping to larger areas. This method can provide a reasonable result of mineral mapping by combining a complete MS image and an incomplete HS image, but it is predicted that this method is sensitive to spectral inconsistency between the HS- and the MS-images, caused by inaccuracies from radiometric calibration and atmospheric correction. Thus in this study, we propose an alternative approach with higher robustness to such spectral inconsistency, based on Kruse and Perry's method (referred to as the K\&P method). First, the K\&P method is reviewed in Sec. 2, and then our method is proposed in Sec. 3. In the following sections, the two methods are evaluated using AVIRIS and ASTER images around Cuprite and Goldfield, Nevada, USA.

\section{Kruse and Perry's Method}

In the K\&P method, spatially nested HS and MS observation images are first acquired, and then converted to surface reflectance images by atmospheric correction. Next, HS-based endmembers for minerals are determined from the HS image, and the spectra of them are weighted by the response functions of the MS sensor bands. Thus the spectra of the MS-based endmembers are obtained, and then used for MS-based mineral mapping with the MS image by the spectral angle mapper (SAM) ${ }^{16}$ or other methods. MS-based mapping results can be compared with HS-based mapping results in the overlapping area. Finally, the MS-based results are extended to the nonoverlapping area using these results.

Though this method derives the MS-based endmember spectra by weighting HS-based endmember spectra with the response functions of the MS sensor bands, it is sensitive to spectral inconsistency between the HS- and the MS images caused by inaccuracies from radiometric calibration and atmospheric correction. For example, if surface reflectance spectra derived from the HS image have spectral distortions causing misclassification of minerals, the MSbased endmember spectra derived from them by weighting will also have such distortions, and they will produce errors in mineral classification with the actual MS image. Even if the HS surface reflectance spectra are accurate, minerals will be unsuccessfully classified if surface reflectance spectra of the MS image have spectral distortions due to calibration and/or atmospheric correction errors.

\section{Proposed Method}

\subsection{Concept and Procedures}

Since the K\&P method is sensitive to inaccuracies from radiometric calibration and/or atmospheric correction, we propose its modified version as described below.

First, the HS- and the MS-images in an overlapping area are accurately co-registered between them and converted to surface reflectance images by atmospheric correction, respectively. Mineral discrimination is then performed with the HS-image using a general procedure like (1) data compression by the minimum noise fraction (MNF) conversion, ${ }^{17}$ (2) extraction of spectrally pure pixels by the pixel purity index (PPI) analysis, ${ }^{18}$ (3) extraction of endmember pixels 


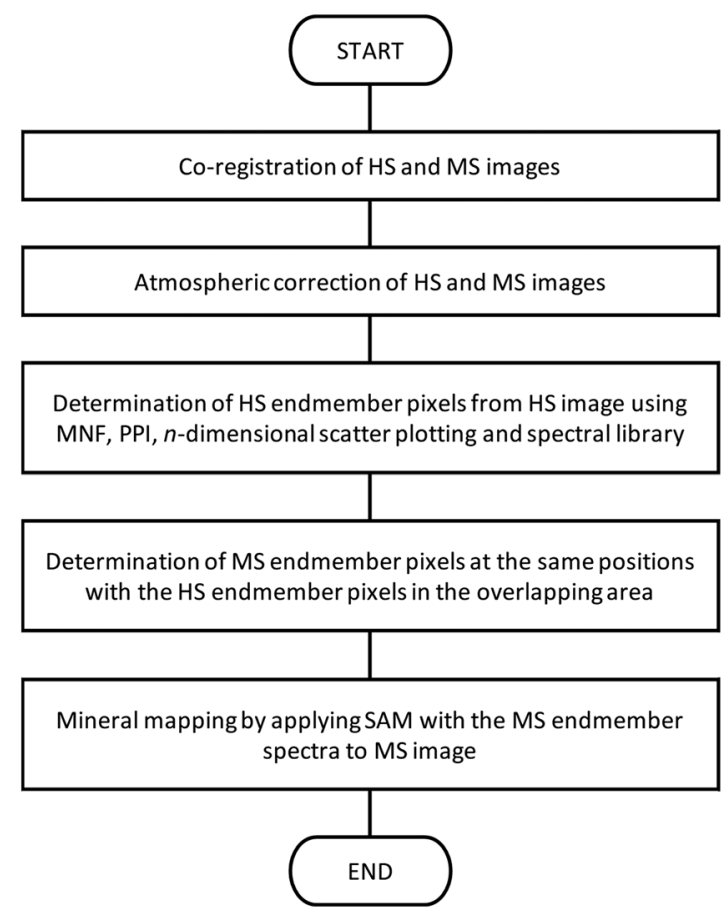

Fig. 1 Flowchart of the proposed method.

by a $n$-dimensional scatter plotting, ${ }^{18}$ and (4) determination of minerals corresponding to each endmember through a comparison between the endmember spectra and the library spectra. This procedure links HS-based endmember spectra and mineral labels in the overlapping area. Next, the MS-based endmember spectra are taken from surface reflectance spectra of the MS-pixels at the same positions with the HS-pixels selected as the HS-based endmembers in the overlapping area. This means that MS-based endmember spectra are linked to mineral labels via HS-based endmember spectra in the overlapping area. Finally, mineral discrimination is performed for the MS-image over the overlapping and the nonoverlapping areas using the MS-based endmember spectra obtained above, assuming that the MS-based endmember spectra can be applied not only to the overlapping area but also to the nonoverlapping area. Figure 1 shows the flowchart of the proposed method.

Though the K\&P method uses the extracted HS-based endmember spectra in calculating the MS-based endmember spectra, the proposed method calculates the MS-based endmember spectra from surface reflectance spectra of the MS-pixels at the same positions with the HSpixels selected as the HS-based endmembers in the overlapping area. Since the MS-based endmember spectra are determined based on the MS-image itself, spectral inconsistency between the HS- and the MS-surface reflectance images will not produce an error in mineral mapping by the SAM method with the MS-image and the MS-based endmember spectra, unlike the K\&P method.

\subsection{Necessity of Accurate Co-registration}

In the proposed method, co-registration between the HS- and the MS-images should be accurately performed, because the MS-based endmembers are derived from the MS-pixels corresponding to the HS-pixels selected as the HS-based endmembers. If co-registration is inaccurate, the proposed method will perform less well, as demonstrated in Sec. 5.4. Therefore, accurate image-to-image matching between the HS- and the MS-images should be applied after system geometric correction of each image. Though various matching methods are available, ${ }^{19}$ its simplest method is probably to match the positions of the two images visually by parallel translation in integer pixels using an appropriate feature point, if geometric distortion is small in each image after system geometric correction. 
If the whole of the overlapping area cannot be co-registered with enough accuracy by single operation, co-registration can be performed for each subarea divided from the whole area.

\subsection{Details of Mineral Mapping with MS-Based Endmember Spectra}

Several methods are available for mineral mapping with the MS-based endmember spectra. In the proposed method, we employed the SAM method for this purpose. The SAM method determines the spectral similarity between two spectra by calculating the angle between the two spectra, treating them as vectors in a space with dimensionality equal to the number of bands, ${ }^{16}$ where the cosine similarity calculated from the angle is often used instead of the angle. In the proposed method, each MS-pixel is assigned to the MS-based endmember that the cosine similarity against that pixel is highest among all the MS-based endmembers and then classified to the mineral corresponding to the assigned MS-based endmember.

In practice, the highest or near-highest cosine similarity can be often found in two or more minerals. In addition, some minerals will often show a lower cosine similarity against a target pixel than other minerals due to spectral distortion even if that mineral is present at that pixel location. The proposed method, therefore, employs a thresholding approach for solving these problems. In this approach, the threshold of the cosine similarity $\left(C_{k}\right)$ is determined for each mineral $k$ in the overlapping area as follows:

(1) If a certain value of $C_{k}$ is given for mineral $k$, the MS-based map of $k$ is obtained by thresholding with that value of $C_{k}$, and its producer's accuracy can be calculated from the obtained MS-based map and the HS-based map of $k$. In addition, the producer's accuracy will increase with decreasing $C_{k}$, because more MS-pixels will be selected for mineral $k$. Thus the maximum value of $C_{k}$ can be searched under the condition that the producer's accuracy exceeds a predefined threshold $P_{0}$ (e.g., 0.9).

(2) For mineral $k$, thresholding with the maximum value of $C_{k}$ obtained above is applied, and the user's accuracy is calculated. If the user's accuracy exceeds a predefined threshold $U_{0}$ (e.g., 0.3 ), this value of $C_{k}$ is used as the threshold for $k$. If not so, the following procedure is performed.

a) The maximum producer's accuracy for $k\left(P_{\max }\right)$ is searched by changing $C_{k}$ under the condition that the user's accuracy for $k$ exceeds a predefined threshold $U_{1}$ (e.g., 0.15 ).

b) The producer's accuracy $P_{1}$ giving the maximum user's accuracy is searched by changing $C_{k}$ under the condition of $P_{1}>P_{\max }-\Delta P$ (e.g., $\Delta P=0.1$ ). The maximum value of $C_{k}$ is searched under the condition that the producer's accuracy exceeds $P_{1}$ and used as the threshold for $k$.

The basic concept of this procedure is to find $C_{k}$ giving an acceptable producer's accuracy for mineral $k$ and to recalculate $C_{k}$ by relaxing the condition of the producer's accuracy if its user's accuracy is not acceptable. Thus the threshold of the cosine similarity is determined for each mineral in the overlapping area, and all minerals that exceed each threshold are selected as candidate minerals for each pixel in the overlapping area.

If two or more minerals remain as candidates for an MS-pixel, the mineral with the highest cosine similarity is basically assigned to that pixel. However, sometimes this mineral is not dominant in that pixel. If such case is observed through comparison between the HS- and the MSmineral maps, the second or subsequent highest mineral in the cosine similarity can be selected instead of the highest mineral for getting higher consistency between the HS- and the MSmineral maps. As a result, the MS-based mineral map obtained will become more consistent with the HS-based mineral map.

\section{Validation Study Using AVIRIS and ASTER Images}

\subsection{Study Areas}

In this paper, AVIRIS and ASTER images acquired around Cuprite and Goldfield, Nevada, USA, were used for validation of the proposed method. Figure 2 shows the locations of these study 


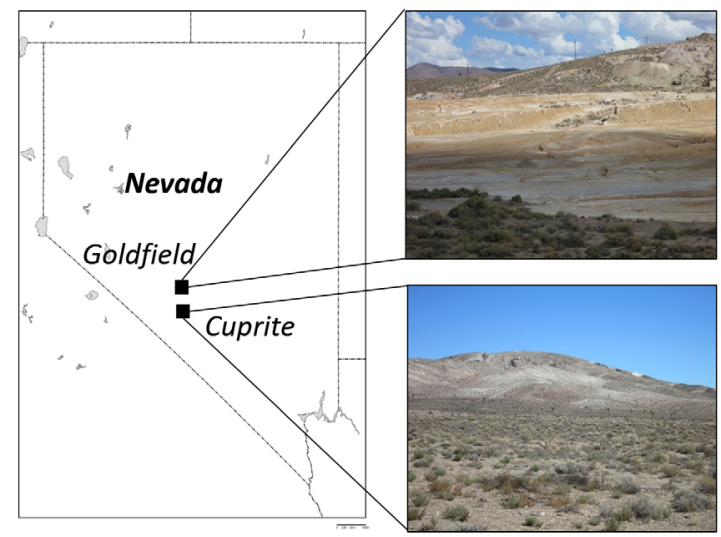

Fig. 2 Locations of the Cuprite and the Goldfield study areas, Nevada, USA. The photos were taken by the authors.
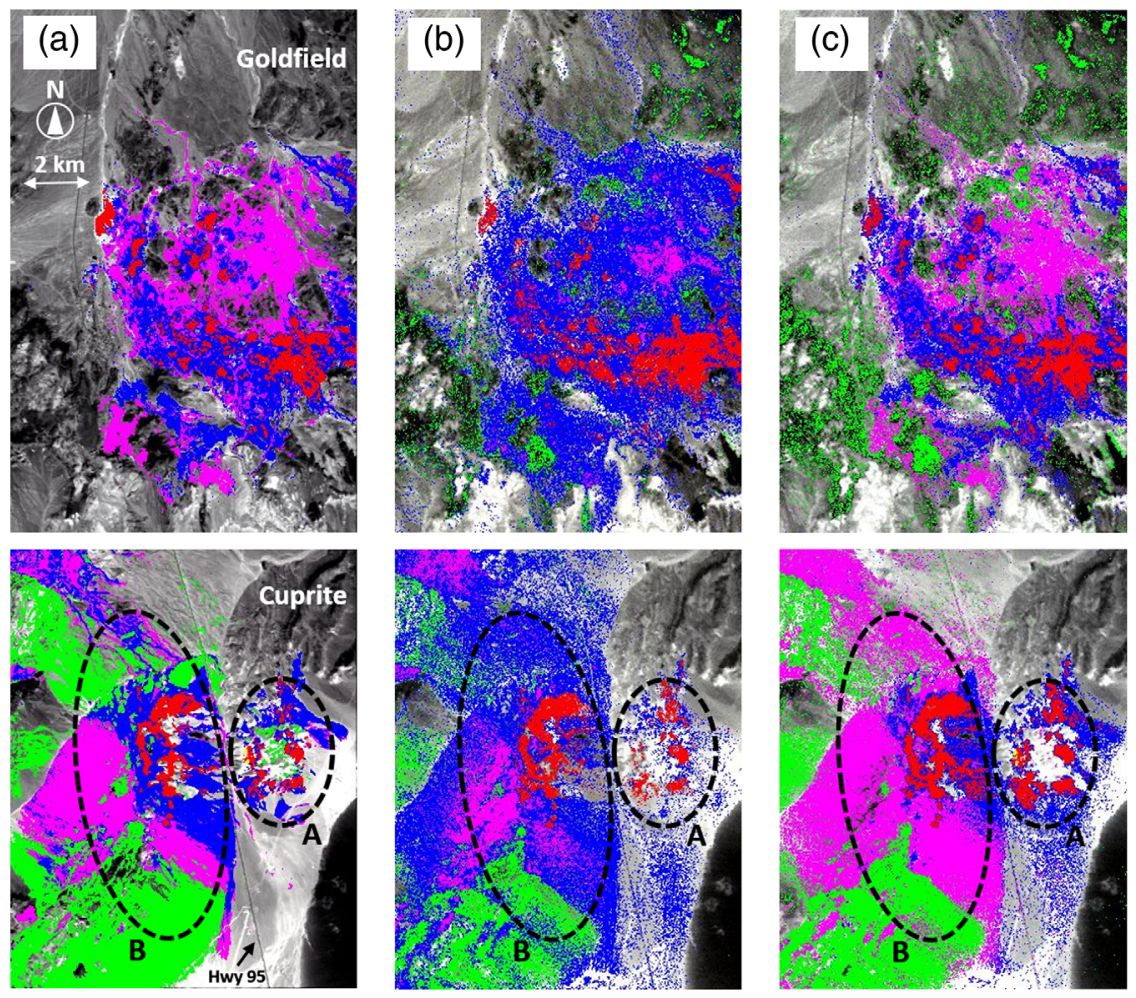

Fig. 3 Mineral maps derived (a) from the AVIRIS image by the traditional way, and from the AST_07XT image (b) by the K\&P method and (c) by the proposed method (blue, kaolinite; red, alunite; pink, muscovite; green, calcite; and yellow, buddingtonite).

areas. These areas are covered by various minerals including silicate minerals such as kaolinite and muscovite, sulfate minerals such as alunite, and carbonate minerals such as calcite. ${ }^{20-23}$ Small amounts of buddingtonite are also seen in the east side of Highway 95 (shown by "A" in Fig. 3) in the Cuprite study area (referred to as the eastern part of Cuprite).

\subsection{Mineral Mapping Using the AVIRIS Image}

The AVIRIS image used was acquired over the study areas on September 20, 2006, with a ground resolution of $15.7 \mathrm{~m}$ (flight name: f060920t01p00r05). The wavelength region used is from 1967 to $2496 \mathrm{~nm}$, because this spectral region allows identification of some important 

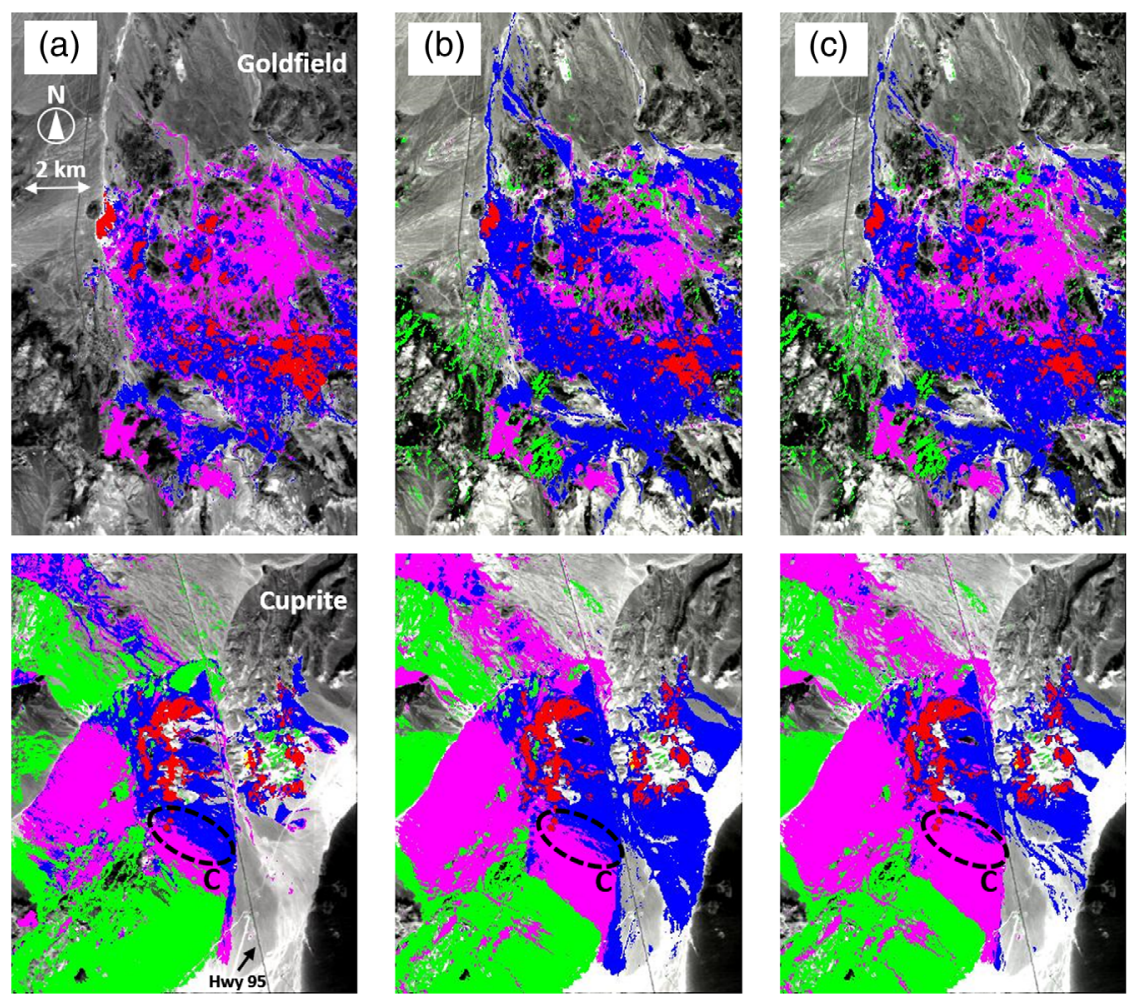

Fig. 4 Mineral maps derived (a) from the AVIRIS image by the traditional way, and from the ASTER simulation image (b) by the K\&P method and (c) by the proposed method (blue, kaolinite; red, alunite; pink, muscovite; green, calcite; and yellow, buddingtonite).

minerals,${ }^{15}$ and also because we compare the results with the published papers on the K\&P method. ${ }^{14,15}$ First, the image was atmospherically corrected by the FLAASH module on the ENVI software ${ }^{24}$ and then converted to the surface reflectance image. Then mineral mapping was performed with this image by the MNF conversion, the PPI analysis, the endmember extraction, and the SAM method implemented in the ENVI software. This processing is referred to as the traditional way from now on.

The result of mineral mapping is displayed by Fig. 3(a), where kaolinite, alunite, muscovite, calcite, and buddingtonite are shown in blue, red, pink, green, and yellow, respectively. This color assignment is used for the following figures in this paper. Unclassified pixels are displayed by the grayscale image of AVIRIS band $171(1976 \mathrm{~nm})$, and this way is also applied to (a) in Figs. 4-6. Figure 3 shows that the eastern part of Cuprite has annularly distributed alunite with surrounding kaolinite, and small amounts of buddingtonite, and the western part of Cuprite (shown by "B" in Fig. 3) has alunite surrounded by kaolinite, muscovite, and calcite. The Goldfield area located in the north of Cuprite is shown to be dominated by muscovite with surrounding kaolinite and alunite. These classification results are visually consistent with previously published studies. ${ }^{22,23}$

\subsection{Mineral Mapping Using the ASTER Image}

The ASTER image used is the shortwave infrared (SWIR) image provided as the ASTER Surface Reflectance VNIR and Crosstalk Corrected SWIR (AST_07XT) product, acquired with a ground resolution of $30 \mathrm{~m}$ over the both study areas on August 15, 2006 (local granule ID: AST_07XT_00308152006183834_20180112005948_22159). Using this ASTER image and the previous AVIRIS image, mineral mapping was performed using the following procedures.

First, the AVIRIS image (15.7-m resolution) and the ASTER image (30-m resolution) were co-registered with resampling to ASTER's pixel resolution $(30 \mathrm{~m})$. In this processing, both 

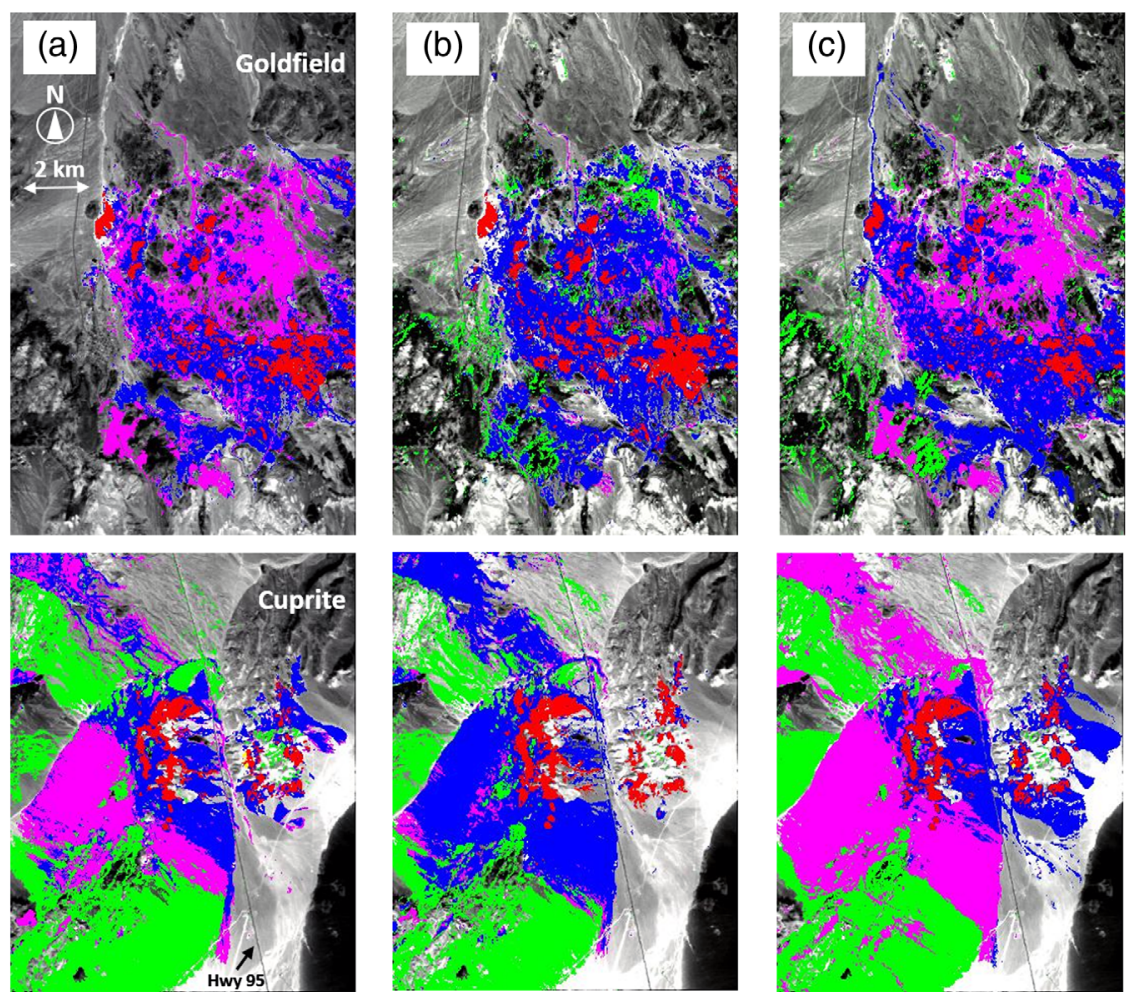

Fig. 5 Mineral maps derived (a) from the AVIRIS image by the traditional way, and from the spectrally modified ASTER simulation image (b) by the K\&P method and (c) by the proposed method (blue, kaolinite; red, alunite; pink, muscovite; green, calcite; and yellow, buddingtonite).
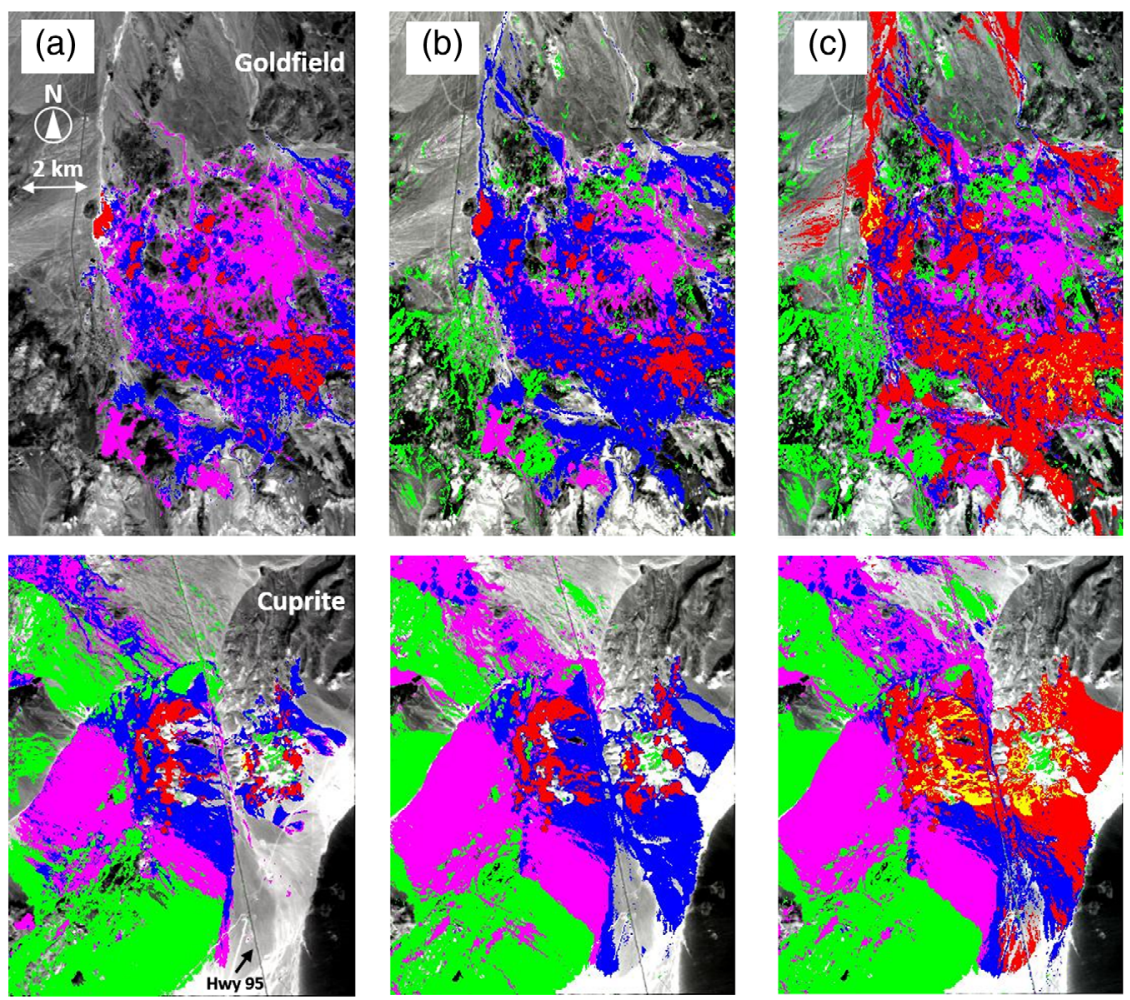

Fig. 6 Mineral maps derived (a) from the AVIRIS image by the traditional way, and from the shifted ASTER simulation image (b) by the K\&P method and (c) by the proposed method (blue, kaolinite; red, alunite; pink, muscovite; green, calcite; and yellow, buddingtonite). 
images were co-registered based on geolocation information attached to each image, and then visually adjusted by parallel translation in integer pixels using a feature point near the center line of the AVIRIS image around the Cuprite study area.

Next, the K\&P and the proposed methods were applied to the pair of the AVIRIS and the ASTER images. In these methods, the Cuprite area was defined as the overlapping area of the HS- and the MS-images, and the Goldfield area was defined as the nonoverlapping area covered by only the MS-image. These definitions are used also in the following sections. The HS-image acquired over the Goldfield study area is used only for evaluating the results of the K\&P and the proposed methods. In the proposed method, MS-based endmember pixels were selected from each subarea divided based on distributions of the HS-based endmembers, where subareas with a co-registration error of one or more pixels were additionally co-registered by parallel translation in integer pixels using a feature point in each subarea. The thresholding method mentioned in Sec. 3.3 was applied not only to the proposed method but also to the K\&P method. In the two methods, alunite was exceptionally assigned to all pixels that alunite remained as one of candidates, resulting in higher consistency with the HS-based mineral map.

Figures 3(b) and 3(c) show the mineral maps obtained by the K\&P and the proposed methods, respectively. Unclassified pixels are displayed by the grayscale image of ASTER/SWIR band 5, and this way is also applied to (b) and (c) in Figures 4 to 6. In Figs. 3(b) and 3(c), kaolinite, muscovite, and calcite have many spike-like noises caused by noises on the ASTER image. The K\&P method misclassified some muscovite as kaolinite and could not detect buddingtonite, probably because of spectral inconsistency between the AVIRIS and the ASTER images, as discussed in Sec. 5.3. On the other hand, the proposed method demonstrates better agreement with the AVIRIS-based map than the K\&P method. For example, the distributions of alunite and kaolinite in the eastern part of Cuprite (shown by "A" in Fig. 3) show similar patterns with the AVIRIS-based map, and buddingtonite exists in only the eastern part of Cuprite, not in the western part of Cuprite (shown by "B" in Fig. 3) and the Goldfield area. In the western part of Cuprite, alunite is surrounded by kaolinite, muscovite, and calcite, as shown in the AVIRISbased map. In the Goldfield area, muscovite and kaolinite are correctly separated, though calcite was partly misclassified. As for a part of the western part of Cuprite, it is known that kaolinite and muscovite occur as mixtures and cannot be easily discriminated even using the AVIRIS image. ${ }^{20,21}$

Table 1 provides the producer's accuracy and the user's accuracy for the K\&P and the proposed methods in the Cuprite and Goldfield areas, assuming that the AVIRIS-based map represents the true distribution of minerals on the ground. In this table, a hyphen for the producer's accuracy means that no pixel was classified as that mineral in the AVIRIS-based map, and a hyphen for the user's accuracy means that no pixel was classified as that mineral by the $\mathrm{K} \& \mathrm{P}$ or the proposed methods.

Table 1 The producer's and the user's accuracies of the K\&P and the proposed methods with the AST_07XT image in the Cuprite and the Goldfield areas for each mineral. Each value in bold face is more than $5 \%$ better than the other.

\begin{tabular}{|c|c|c|c|c|c|c|c|c|}
\hline & \multicolumn{4}{|c|}{ Cuprite area } & \multicolumn{4}{|c|}{ Goldfield area } \\
\hline & \multicolumn{2}{|c|}{$\begin{array}{c}\text { Producer's } \\
\text { accuracy (\%) }\end{array}$} & \multicolumn{2}{|c|}{$\begin{array}{c}\text { User's accuracy } \\
(\%)\end{array}$} & \multicolumn{2}{|c|}{$\begin{array}{c}\text { Producer's } \\
\text { accuracy (\%) }\end{array}$} & \multicolumn{2}{|c|}{$\begin{array}{c}\text { User's accuracy } \\
(\%)\end{array}$} \\
\hline & $K \& P$ & Proposed & $K \& P$ & Proposed & $\mathrm{K} \& \mathrm{P}$ & Proposed & $\mathrm{K} \& \mathrm{P}$ & Proposed \\
\hline Kaolinite & 58.6 & 34.9 & 19.4 & 39.1 & 63.9 & 60.2 & 25.4 & 42.7 \\
\hline Alunite & 65.0 & 80.0 & 51.0 & 47.0 & 59.4 & 57.9 & 39.9 & 48.4 \\
\hline Muscovite & 35.0 & 79.7 & 67.7 & 42.9 & 11.8 & 48.9 & 71.8 & 50.5 \\
\hline Calcite & 38.0 & 55.1 & 77.3 & 75.6 & - & - & 0.0 & 0.0 \\
\hline Buddingtonite & 0.0 & 36.1 & - & 22.4 & - & - & - & - \\
\hline
\end{tabular}


As for muscovite, the producer's accuracy is better in the proposed method, and the user's accuracy is better in the K\&P method. As for calcite, the producer's accuracy is much better in the proposed method, whereas the user's accuracy is a little better in the K\&P method. Buddingtonite in the Cuprite area was detected by the proposed method partly, but not by the K\&P method. The producer's accuracy and the user's accuracy for alunite are almost same between the two methods. As for kaolinite, the producer's accuracy is better in the K\&P method, and the user's accuracy is better in the proposed method.

\section{Performance Analysis Using the ASTER Simulation Image}

\subsection{Overview}

It is expected that the proposed method is less sensitive to inaccuracies from radiometric calibration and atmospheric correction than the K\&P method. In order to confirm this, we evaluated the two methods using an ASTER simulation image. This image was generated by weighting the AVIRIS surface reflectance image with the response functions of ASTER's SWIR bands (bands 4 to 9). Using the ASTER simulation image, the two methods can be evaluated without geometric or radiometric errors.

\subsection{Mineral Mapping Using the ASTER Simulation Image}

The K\&P and the proposed methods were applied to the AVIRIS image and the ASTER simulation image. The definitions of the overlapping and the nonoverlapping areas were same with Sec. 4.3. The thresholding of the cosine similarity with the exceptional assignment of alunite was applied to the two methods in the same way as Sec. 4.3.

Figure 4 displays the mineral maps obtained from the AVIRIS image and from the ASTER simulation image by each method, showing similar distributions of minerals between the two methods. Table 2 provides the producer's accuracy and the user's accuracy for each method for the Cuprite and the Goldfield areas, assuming that the AVIRIS-based map represents the true distribution of minerals on the ground. In the Cuprite area, the K\&P and the proposed methods show the producer's accuracy of about $90 \%$ for alunite and muscovite, though showing the user's accuracy of about $50 \%$ for muscovite. As for kaolinite, the producer's accuracy and the user's accuracy decreased to $50 \%$ or $60 \%$ in the two methods, mainly because some of kaolinite in the western part of Cuprite (shown by "C" in Fig. 4) were misclassified as muscovite. Since kaolinite and muscovite occur as mixtures around the area "C," ${ }^{, 20,21}$ it is not easy to discriminate these minerals even by AVIRIS-based mineral mapping, so that the producer's accuracy decreased by a small difference in the cosine similarity between kaolinite and muscovite,

Table 2 The producer's and the user's accuracies of the K\&P and the proposed methods with the ASTER simulation image in the Cuprite and the Goldfield areas for each mineral. Each value in bold face is more than $5 \%$ better than the other.

\begin{tabular}{|c|c|c|c|c|c|c|c|c|}
\hline & \multicolumn{4}{|c|}{ Cuprite area } & \multicolumn{4}{|c|}{ Goldfield area } \\
\hline & \multicolumn{2}{|c|}{$\begin{array}{c}\text { Producer's } \\
\text { accuracy (\%) }\end{array}$} & \multicolumn{2}{|c|}{$\begin{array}{c}\text { User's accuracy } \\
(\%)\end{array}$} & \multicolumn{2}{|c|}{$\begin{array}{c}\text { Producer's } \\
\text { accuracy (\%) }\end{array}$} & \multicolumn{2}{|c|}{$\begin{array}{c}\text { User's accuracy } \\
(\%)\end{array}$} \\
\hline & $K \& P$ & Proposed & $K \& P$ & Proposed & $K \& P$ & Proposed & $K \& P$ & Proposed \\
\hline Kaolinite & 63.8 & 47.9 & 44.4 & 49.6 & 93.3 & 87.9 & 52.9 & 62.2 \\
\hline Alunite & 92.0 & 92.0 & 79.2 & 79.2 & 61.0 & 61.0 & 94.5 & 94.5 \\
\hline Muscovite & 87.1 & 90.5 & 53.9 & 48.4 & 56.9 & 71.5 & 90.5 & 86.5 \\
\hline Calcite & 71.7 & 68.9 & 82.4 & 81.8 & - & - & 0.0 & 0.0 \\
\hline Buddingtonite & 80.6 & 80.6 & 90.6 & 90.6 & - & - & - & - \\
\hline
\end{tabular}


particularly in the proposed method. On the other hand, the both methods performed well for buddingtonite and calcite-particularly buddingtonite seen in only the eastern part of Cuprite shows the producer's accuracy of about $80 \%$ and the user's accuracy of about $90 \%$. In the Goldfield area, the producer's accuracy for calcite and buddingtonite was not calculated because they were not detected in AVIRIS-based mineral mapping, though calcite was incorrectly detected and the user's accuracy for it is $0.0 \%$ in the two methods. Muscovite, alunite, and kaolinite were successfully discriminated by each method.

\subsection{Mineral Mapping Using the Spectrally Modified ASTER Simulation Image}

For investigation of sensitivity of each method to spectral distortion in the MS image, the spectrally modified ASTER simulation image which was spectrally consistent with the AST_07XT image used in Sec. 4.3 for each mineral and was consistent with the AVIRIS image except for spectral bands and spectral distortion was generated as follows. First, the surface reflectance ratio of the AST_07XT-based endmember (Sec. 4.3) to the ASTER simulation-based endmember (Sec. 5.2) was calculated at each band for each mineral. Then the ratio of each band was averaged over all minerals and then multiplied to the surface reflectance of the ASTER simulation image for each band. Applying the K\&P and the proposed methods to this spectrally modified ASTER simulation image, we can evaluate the influence of spectral distortion for each method.

Figure 5 shows the mineral maps obtained from the AVIRIS image and the spectrally modified ASTER simulation image by each method, and Table 3 gives the producer's accuracy and the user's accuracy for each case, assuming that the AVIRIS-based map represents the true distribution of minerals on the ground. The thresholding of the cosine similarity with the exceptional assignment of alunite was applied to the two methods in the same way as Sec. 4.3. As shown, the K\&P method failed to classify muscovite and kaolinite correctly, as described by Kruse et al. ${ }^{6}$ Particularly, the producer's accuracy for muscovite is much lower in the K\&P method than in the proposed method, because muscovite and kaolinite had similar spectra due to spectral distortion and the K\&P method is sensitive to such error factor. The reason why the K\&P method gives higher producer's accuracy for kaolinite than the proposed method is because the proposed method classified kaolinite as muscovite in the mixed area of them (C in Fig. 4) - actually, the user's accuracy for kaolinite is lower in the K\&P method than in the proposed method. The K\&P method failed to detect buddingtonite, because the threshold used in the SAM method could not be determined for buddingtonite. Calcite and alunite show similar performance in the two methods, because the spectra of these minerals were not strongly affected by spectral distortion due to their unique shapes. As a whole, the proposed method shows more consistent results with the AVIRIS image, which indicates that the proposed method is more robust against spectral distortion than the K\&P method.

Table 3 The producer's and the user's accuracies of the K\&P and the proposed methods with the spectrally modified ASTER simulation image in the Cuprite and the Goldfield areas for each mineral. Each value in bold face is more than $5 \%$ better than the other.

\begin{tabular}{|c|c|c|c|c|c|c|c|c|}
\hline & \multicolumn{4}{|c|}{ Cuprite area } & \multicolumn{4}{|c|}{ Goldfield area } \\
\hline & \multicolumn{2}{|c|}{$\begin{array}{c}\text { Producer's } \\
\text { accuracy (\%) }\end{array}$} & \multicolumn{2}{|c|}{$\begin{array}{c}\text { User's accuracy } \\
(\%)\end{array}$} & \multicolumn{2}{|c|}{$\begin{array}{c}\text { Producer's } \\
\text { accuracy (\%) }\end{array}$} & \multicolumn{2}{|c|}{$\begin{array}{c}\text { User's accuracy } \\
(\%)\end{array}$} \\
\hline & $K \& P$ & Proposed & $K \& P$ & Proposed & $K \& P$ & Proposed & $K \& P$ & Proposed \\
\hline Kaolinite & 68.4 & 47.3 & 38.1 & 56.5 & 71.2 & 86.4 & 46.3 & 65.5 \\
\hline Alunite & 92.0 & 92.0 & 63.2 & 78.2 & 97.0 & 63.5 & 73.6 & 94.1 \\
\hline Muscovite & 10.9 & 90.4 & 49.1 & 49.3 & 11.8 & 72.6 & 82.9 & 86.0 \\
\hline Calcite & 73.7 & 71.3 & 91.0 & 80.8 & - & - & 0.0 & 0.0 \\
\hline Buddingtonite & 0.0 & 80.6 & - & 90.6 & - & - & - & - \\
\hline
\end{tabular}




\subsection{Mineral Mapping Using the Shifted ASTER Simulation Image}

For investigation of sensitivity of each method to co-registration errors between the HS- and the MS-images, the ASTER simulation image was shifted by +2 pixels toward the right and +2 pixels toward the upper. This shifted ASTER simulation image is consistent with the AVIRIS image except for spectral bands and pixel positions. In calculation of the producer's accuracy and the user's accuracy, the shifted ASTER simulation image was shifted back to the original position of the AVIRIS image.

Figure 6 shows the mineral maps obtained from the AVIRIS image and the shifted ASTER simulation image by each method, and Table 4 gives the producer's accuracy and the user's accuracy for each case, assuming that the AVIRIS-based map represents the true distribution of minerals on the ground. The thresholding of the cosine similarity with the exceptional assignment of alunite was applied to the two methods in the same way as Sec. 4.3. As shown, the results by the K\&P method are almost same with those for the ASTER simulation image (Sec. 5.2), supporting our prediction that the K\&P method should be robust against co-registration errors in

Table 4 The producer's and the user's accuracies of the K\&P and the proposed methods with the shifted ASTER simulation image in the Cuprite and the Goldfield areas for each mineral. Each value in bold face is more than $5 \%$ better than the other.

\begin{tabular}{|c|c|c|c|c|c|c|c|c|}
\hline & \multicolumn{4}{|c|}{ Cuprite area } & \multicolumn{4}{|c|}{ Goldfield area } \\
\hline & \multicolumn{2}{|c|}{$\begin{array}{l}\text { Producer's } \\
\text { accuracy (\%) }\end{array}$} & \multicolumn{2}{|c|}{$\begin{array}{c}\text { User's accuracy } \\
(\%)\end{array}$} & \multicolumn{2}{|c|}{$\begin{array}{l}\text { Producer's } \\
\text { accuracy (\%) }\end{array}$} & \multicolumn{2}{|c|}{$\begin{array}{c}\text { User's accuracy } \\
(\%)\end{array}$} \\
\hline & $K \& P$ & Proposed & $K \& P$ & Proposed & $K \& P$ & Proposed & $K \& P$ & Proposed \\
\hline Kaolinite & 58.8 & 25.6 & 43.9 & 33.3 & 91.5 & 21.3 & 55.0 & 25.9 \\
\hline Alunite & 98.1 & 61.6 & 64.4 & 11.3 & 80.5 & 87.2 & 87.8 & 15.4 \\
\hline Muscovite & 87.6 & 86.2 & 52.4 & 49.9 & 58.5 & 55.5 & 89.0 & 70.2 \\
\hline Calcite & 72.8 & 77.1 & 77.2 & 73.3 & - & - & 0.0 & 0.0 \\
\hline Buddingtonite & 88.9 & 44.4 & 69.6 & 0.5 & - & - & - & - \\
\hline
\end{tabular}
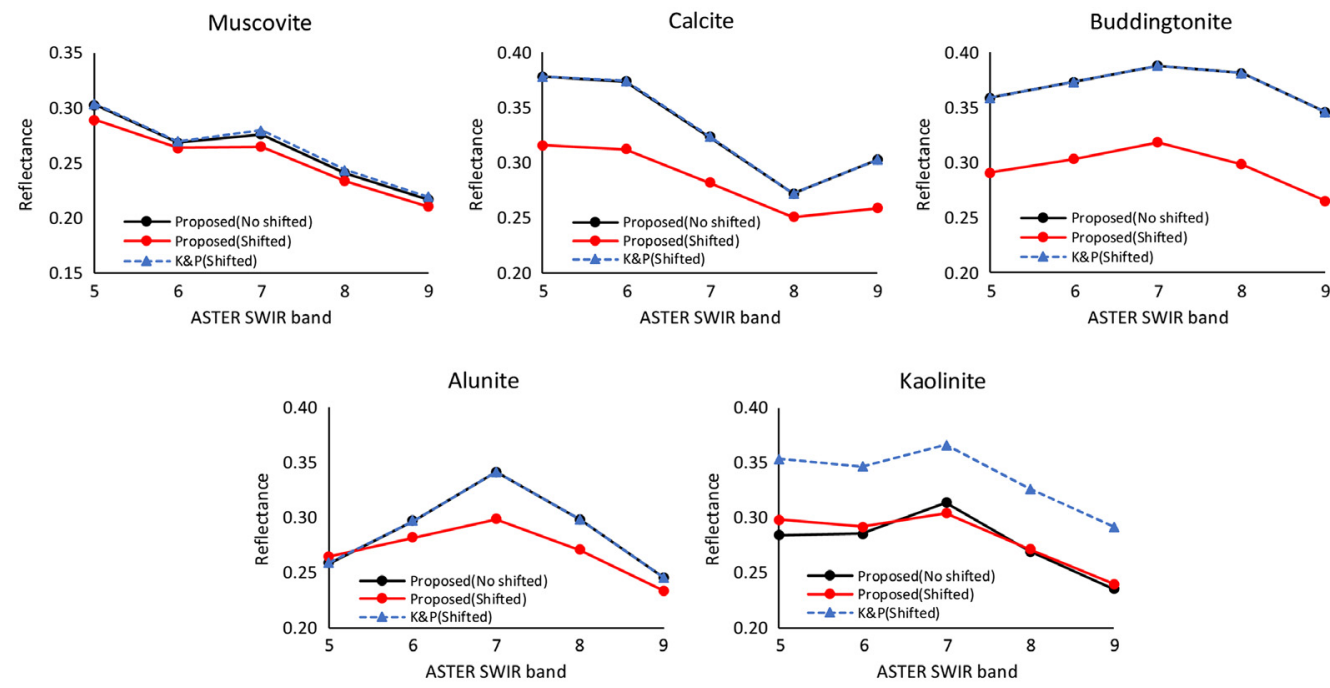

Fig. 7 Comparison of the endmember spectra obtained from the ASTER simulation image (no shifted) and the shifted one by the proposed method, and from the shifted one by the K\&P method, for each mineral. 
principle, though these results are dependent on homogeneity of the study areas to some extent. On the other hand, the proposed method performed well for muscovite and calcite, but less well for buddingtonite, alunite, and kaolinite in the two areas. For example, most of areas classified as kaolinite in the ASTER simulation image (Sec. 5.2) are misclassified as alunite. Also a part of areas classified as alunite and kaolinite in the Cuprite area are misclassified as buddingtonite. Thus the producer's accuracies for buddingtonite and kaolinite reduced almost by half, and the user's accuracy for buddingtonite is just $0.5 \%$. The accuracies for alunite also decreased, though only the producer's accuracy in the Goldfield area increased.

The reason why the proposed method performed well in muscovite and calcite but less well in buddingtonite, alunite, and kaolinite is because the endmember spectra of the latter minerals are not consistent between the ASTER simulation image and the shifted one due to spatial distributions that involve widely separated exposures of small areal extent, unlike the former minerals, as shown by Fig. 7. It must, therefore, be recognized that the proposed method is sensitive to coregistration errors for minerals with such spatial distributions. In the case of the K\&P method, the ASTER simulation image and the shifted one provided almost same spectra for all minerals. The spectra obtained from the shifted one by the K\&P method are displayed in Fig. 7 for comparison to the spectra of the proposed method.

\section{Discussion}

Figure 8 summarizes the producer's accuracy and the user's accuracy of each mineral in the four cases shown by Tables 1 to 4: AST_07XT, the ASTER simulation image (AST_sim), the spectrally modified ASTER simulation image (AST_sim+sp), and the shifted ASTER simulation image (AST_sim+shift). In this figure, zero is assigned to the user's accuracy of the K\&P method for Buddingtonite in AST_07XT and AST_sim+sp, because the K\&P method could not select any pixels for that mineral from these images. This figure indicates the following.

- As a whole, the producer's accuracy is somewhat higher than the user's accuracy, because the producer's accuracy was prioritized in thresholding of the cosine similarity.

- The producer's accuracy for AST_07XT is higher in the proposed method than in the K\&P method as a whole. In addition, the producer's accuracy for AST_sim is higher than that for AST_07XT in the two methods, particularly in the K\&P method. These indicate that AST_07XT is somewhat spectrally inconsistent with the AVIRIS image, and it decreased the producer's accuracy for AST_07XT in the K\&P method, while it gave almost no impact to the proposed method. The fact that the K\&P method is sensitive to spectral inconsistency between HS- and MS-images is demonstrated also by the results of AST_sim+sp.

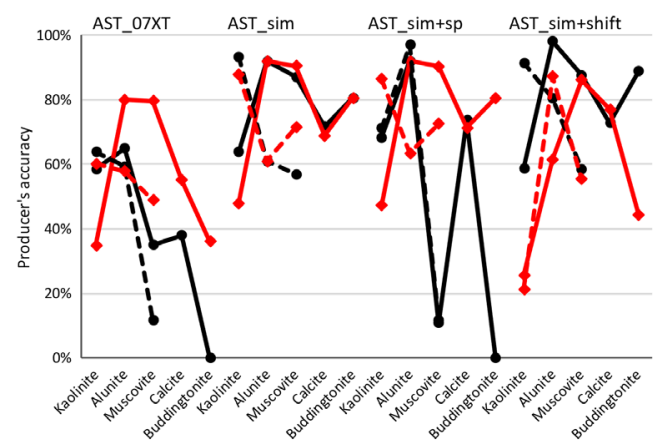

(a)

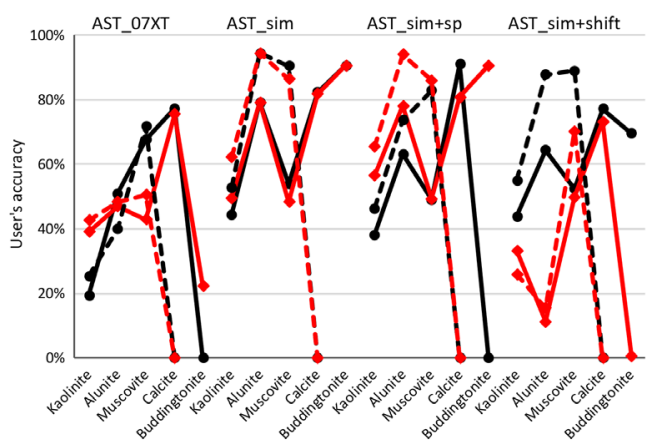

(b)

Fig. 8 Comparisons of (a) the producer's accuracy and (b) the user's accuracy derived by the K\&P method (black circle) and the proposed method (red diamond) in the Cuprite study area (solid line) and the Goldfield study area (dashed line) for the AST_07XT image, the ASTER simulation image (AST_sim), the spectrally modified ASTER simulation image (AST_sim+sp), and the shifted ASTER simulation image (AST_sim+shift). 
- The proposed method shows similar performance for AST_07XT, AST_sim, and AST_sim+sp, though the user's accuracy for AST_07XT is somewhat low. This indicates that the proposed method is robust against spectral inconsistency between HS- and MSimages. On the other hand, the results of AST_sim+shift show that the proposed method is sensitive to co-registration errors. Also the reason why the proposed method performed less well in AST_07XT than in AST_sim even though the method is robust against spectral inconsistency will be partly because AST_07XT contains a co-registration error. However, the impact of co-registration errors in the proposed method is not significant if a mineral distributes homogeneously in a wide area, as supported by the fact that the proposed method showed similar performance between AST_sim and AST_sim+shift for muscovite and calcite.

According to the above, it can be concluded as follows.

If HS-MS image matching can be performed accurately enough, or if an applied area is highly homogeneous in mineral distributions, the proposed method has an advantage in robustness. The result of AST_sim+shift indicates that the proposed method was degraded even by co-registration errors of two pixels. Thus the following preprocessing should be required for the proposed method: first, system geometric correction is applied to each of HS and MS images, and then image-to-image matching with an accuracy of one pixel or less is applied to these images. Accurate image matching methods like the phase-only correlation method ${ }^{25}$ are available for this purpose.

On the other hand, if HS-MS image matching cannot be performed with enough accuracy like for airborne images, or if an applied area contains minerals with spatial distributions that involve widely separated exposures of small areal extent, the K\&P method has an advantage. In applying the K\&P method, HS- and MS-images should be radiometrically corrected by accurate radiometric calibration and atmospheric correction, and also accurate matching of spectral reflectance between those images is desirable. It may be required that long-term and short-term changes in radiometric calibration coefficients are considered for accurate radiometric calibration, and accurate atmospheric parameters such as aerosol density/visibility/type and water content are obtained for accurate atmospheric correction.

In both methods, a possible common issue is on representativeness of HS-based endmembers obtained from an overlapping area. Since these methods assume that these endmembers can be applied to a nonoverlapping area without deficiency, a lack of HS-based endmembers will cause misdiscrimination of minerals. Thus care must be taken in this regard when applying either the $\mathrm{K} \& \mathrm{P}$ method or the proposed method in the mapping of endmembers.

\section{Summary and Conclusions}

HS imagers are more effective than MS imagers in mineral mapping, but spatial coverage of HS images is limited in comparison to that of MS images. Thus Kruse and Perry have proposed a method that uses coincident HS imaging and MS imaging data to extend mineral mapping to larger areas. ${ }^{14,15}$ In this paper, we proposed a method modified from the K\&P method. Though the K\&P method uses the extracted HS-based endmember spectra in calculating the MS-based endmember spectra, the proposed method calculates the MS-based endmember spectra from surface reflectance spectra of the MS pixels at the same positions with the HS pixels selected as the HS-based endmembers. The validation study using AVIRIS and ASTER images over Cuprite and Goldfield areas, Nevada, USA, demonstrated that the proposed method was more robust against spectral inconsistency between the HS- and the MS-images caused by calibration and/or atmospheric correction errors than the K\&P method, though the proposed method was more sensitive to co-registration errors between the HS- and the MS-images for minerals with spatial distributions that involve widely separated exposures of small areal extent (buddingtonite, alunite, and kaolinite in the validation study) than the K\&P method. The proposed method will, therefore, give more reasonable results if HS-MS image matching can be performed accurately enough, or if an applied area is highly homogeneous in mineral distributions.

The HISUI developed by the Japanese Government will be delivered to the ISS in the near future. ${ }^{13}$ Since HISUI has a narrow swath width of $30 \mathrm{~km}$ and no pointing function, its coverage 
Hirai and Tonooka: Mineral discrimination by combination of multispectral image...

is predicted to be patchy. The proposed method is, therefore, expected to be used for compensating HISUI's observation gaps by combining images of MS sensors such as ASTER.

\section{Acknowledgments}

The authors would like to thank the editor and three reviewers for their comments and suggestions that greatly improved this manuscript.

\section{References}

1. Y. Yamaguchi and I. Takeda, "Mineralogical mapping by ASTER in Cuprite, Nevada, U.S.A.," Asian J. Geoinf. 3(3), 17-24 (2003).

2. Y. Yamaguchi and C. Naito, "Spectral indices for lithologic discrimination and mapping by using the ASTER SWIR bands," Int. J. Remote Sens. 24(22), 4311-4323 (2003).

3. L. C. Rowan and J. C. Mars, "Lithologic mapping in the mountain pass, California area using advanced spaceborne thermal emission and reflection radiometer (ASTER) data," Remote Sens. Environ. 84, 350-366 (2003).

4. S. Tanaka et al., "Mineral discrimination with ASTER data using spectral pattern analysis and forecast of the accuracy evaluation," J. Remote Sens. Jpn. 19(5), 382-406 (1999).

5. F. A. Kruse, W. M. Baugh, and S. L. Perry, "Validation of DigitalGlobe WorldView-3 Earth imaging satellite shortwave infrared bands for mineral mapping," J. Appl. Remote Sens. 9(1), 096044 (2015).

6. F. A. Kruse, S. L. Perry, and A. Caballero, "District-level mineral survey using airborne hyperspectral data, Los Menucos, Argentina," Ann. Geophys. 49(1), 83-92 (2006).

7. F. van der Meer et al., "Wavelength feature mapping as a proxy to mineral chemistry for investigating geologic systems: an example from the Rodalquilar epithermal system," Int. J. Appl. Earth Obs. Geoinf. 64, 237-248 (2018).

8. G. Kereszturi et al., "Integrating airborne hyperspectral imagery and LiDAR for volcano mapping and monitoring through image classification," Int. J. Appl. Earth Obs. Geoinf. 73, 323-339 (2018).

9. L. Liu et al., "Mapping alteration using imagery from the Tiangong-1 hyperspectral spaceborne system: Example for the Jintanzi gold province, China," Int. J. Appl. Earth Obs. Geoinf. 64, 275-286 (2018).

10. M. A. Folkman et al., "EO-1/Hyperion hyperspectral imager design, development, characterization, and calibration," Proc. SPIE 4151, 40-51 (2001).

11. L. Guanter et al., "The EnMAP spaceborne imaging spectroscopy mission for Earth observation," Remote Sens. 7(7), 8830-8857 (2015).

12. P. Stefano et al., "The PRISMA hyperspectral mission: science activities and opportunities for agriculture and land monitoring," in Proc. IEEE Int. Geosci. and Remote Sens. Symp. (IGARSS), pp. 4558-4561 (2013).

13. T. Matsunaga et al., "Current status of hyperspectral imager suite (HISUI) onboard International Space Station (ISS)," in Proc. IEEE Int. Geosci. and Remote Sens. Symp. (IGARSS), pp. 443-446 (2017).

14. F. A. Kruse and S. L. Perry, "Regional mineral mapping by extending hyperspectral signatures using multispectral data," in Proc. IEEE Aerosp. Conf. (2007).

15. F. A. Kruse and S. L. Perry, "Improving multispectral mapping by spectral modeling with hyperspectral signatures," J. Appl. Remote Sens. 3, 033504 (2009).

16. F. A. Kruse et al., "The spectral image processing system (SIPS) - interactive visualization and analysis of imaging spectrometer data," Remote Sens. of Environ. 44, 145-163 (1993).

17. A. A. Green et al., "A transformation for ordering multispectral data in terms of image quality with implications for noise removal," IEEE Trans. Geosci. Remote Sens. 26(1), 65-74 (1988).

18. J. W. Boardman, F. A. Kruse, and R. O. Green, "Mapping target signatures via partial unmixing of AVIRIS data," in Summaries Proc. 5th JPL Airborne Earth Sci. Workshop, JPL Publication, pp. 95-101 (1995). 
19. L. Moigne, N. Netanyahu, and R. Eastman (Eds.), Image Registration for Remote Sensing, Cambridge University Press, Cambridge (2011).

20. R. N. Clark et al., "Imaging spectroscopy: earth and planetary remote sensing with the USGS Tetracorder and expert systems," J. Geophys. Res. 108(E12), 5131 (2003).

21. G. A. Swayze et al., "Mapping advanced argillic alteration at Cuprite, Nevada using imaging spectroscopy," Econ. Geol. 109(5), 1179-1221 (2014).

22. B. W. Rockwell, "The Goldfield mining district, Nevada: an acid-sulfate Bonanza gold deposit," in Proc. 14th Int. Conf. for Appl. Geologic Remote Sens. (2000).

23. B. W. Rockwell, L. C. Bonham, and P. D. Denning, "USGS national map of surficial mineralogy: a new interactive Web resource for the detection, mapping, and mineralogical characterization of hydrothermal alteration and mine waste," in Proc. 17th Annu. Work Digital Mapping Tech. (2013).

24. Exelis Visual Information Solutions, Inc., ENVI Classic Tutorial: Atmospherically Correcting Multispectral Data Using FLAASH ${ }^{\circledR}$, Exelis Visual Information Solutions, Inc., Boulder (2013).

25. K. Takita et al., "High-accuracy image registration based on phase-only correlation," IEICE Trans. Fundam. E86-A(8), 1925-1934 (2003).

Akihiro Hirai received his master's degree in computer and information scie degree in computer and information sciences from Ibaraki University, Ibaraki, Japan, in 2019 and joined Mitsubishi Space Software Co., Ltd., Tokyo. His research interest is in geological remote sensing using hyperspectral imagers.

Hideyuki Tonooka received his BEng, MEng, and DEng degrees in geosystem engineering from the University of Tokyo in 1992, 1994, and 2000, respectively. He is a professor at Ibaraki University, Japan. He has been a member of the Project Science Team for the Advanced Spaceborne Thermal Emission and Reflection Radiometer, part of NASA's Earth Observing System since 1995, and is also joining the Compact Infrared Camera Project of the Japan Aerospace Exploration Agency. His main research activities are in calibration, atmospheric correction, and applications in remote sensing, particularly in the thermal infrared spectral region. 